\title{
Boosting the Efficiency of Suspended Photocatalysts for Overall Water Splitting
}

T $t$ is a significant task to cover the residential energy demand Lof a society (approximately $20 \%$ of the total) with solar energy, but it is a much greater challenge to cover all of it, including the demands of industry, commerce, and transportation. The $20 \%$ goal may be achievable through photovoltaic-powered households and appliances, but the $100 \%$ goal will require extensive facilities for storing, converting, and distributing large quantities of electrons. The necessary batteries and an expanded electrical grid do add to the cost of photovoltaics and make solar energy technology even less competitive with polluting fossil energy carriers.

Solar-energy-driven water electrolysis can be a viable solution to this problem. Hydrogen is the smallest and easiest to fabricate "battery" that exists, and it can be used as a gaseous fuel or as an electron-rich precursor to liquid transportation fuels. The technology for creating hydrogen by oxidation of water (solar water splitting) already exists in the form of multijunction photovoltaic systems coupled to water electrolyzers. These can achieve over $18 \%$ overall efficiency, but they are so expensive that nobody will use them, especially not anyone living on less than $\$ 10$ a day, that is, the majority of people in the world.

To make solar hydrogen affordable for everyone, and to have it outcompete fossil fuels, the cost of water photoelectrolysis needs to be reduced 100 -fold. Photoelectrochemical cells that combine photovoltaic and electrolytic functions in one unit are a step in that direction. ${ }^{1}$ Such "Artificial Leafs" can reach efficiencies of up to $12.4 \%$, ${ }^{2}$ but these devices are still too fragile and too expensive for widespread use. Another promising technology consists of miniaturized photocatalysts that generate hydrogen and oxygen upon exposure of a catalystwater mixture to sunlight. For these catalysts, the term "photochemical diodes" was coined by Arthur Nozik many years ago. ${ }^{3}$ Because of the total integration of components for light absorption, charge separation, and water electrolysis, such systems can be almost 1 order of magnitude cheaper than photoelectrochemical cells at equal efficiency. ${ }^{4,5}$ Many examples of such photocatalysts are known today, ${ }^{6-10}$ but their solar to fuel conversion efficiencies are 3 orders of magnitude too low for practical application. Also, there are concerns about the safe coevolution of hydrogen and oxygen in the same sample space, but this problem can be minimized through reactor compartmentalization and early gas separation.

A guide to optimization of water splitting catalysts can be found in the equivalent circuit diagram for a photovoltaic cell (Figure 1). In order to generate the free energy for water electrolysis, a photocatalyst must be able to generate long-lived charge carriers under sunlight illumination (large generation current $\left.I_{\mathrm{L}}\right)$, it must be able to separate them into electrons and holes (large diode current $I_{\mathrm{D}}$, large shunt resistance $R_{\mathrm{SH}}$ ), and it must be able to quickly transfer them to water (small serial resistance $\left.R_{S}\right) .{ }^{11}$ Suspended microscale powders have an advantage here over conventional photoelectrodes because charge transport pathways from the interior to the solid-water

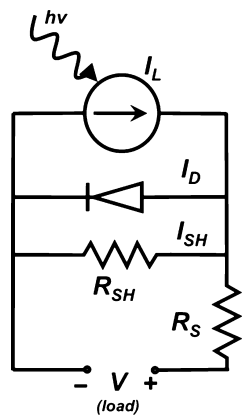

Figure 1. Equivalent circuit diagram for a photovoltaic cell. $R_{\mathrm{SH}}$ : Shunt resistance. $R_{\mathrm{S}}$ : Serial resistance. $I_{\mathrm{SH}}$ : Leakage current. $I_{\mathrm{L}}$ : Generation current. $I_{\mathrm{D}}$ : Diode current.

interface are short, and the large surface area promotes redox reactions with water (which reduces the series resistance $R_{\mathrm{S}}$ ). At the same time, this large interface promotes reverse charge transfer and recombination, which shunt the system (small $R_{\mathrm{SH}}$ in Figure 1), and dissipates the photochemical energy as heat. Also, the rectifying (charge separating) function of the photodiode is diminished in fine powders because the electric field that forms inside the particle (band bending) is limited by the particle size. Accordingly, the diode current $I_{\mathrm{D}}$ is small.

These issues reduce the free energy that can be harvested from illuminated semiconductor particle suspensions. In order for solar hydrogen from inexpensive suspension catalysts to become a reality, researchers must minimize these loss mechanisms in microscale photodiodes.

A. They must develop inexpensive methods to prepare recombination-resistant interfaces and surfaces. In photovoltaics, expensive vacuum deposition methods are often necessary to prepare defect-free interfaces that reduce interfacial recombination. For photocatalysts, analogous but inexpensive and scalable methods for the deposition of "buffer" layers should be developed also. The purpose of these buffer layers is to eliminate midgap states at the surface that cause recombination. Examples for such treatments include fluoride termination for silicon, ${ }^{12}$ and the application of $\mathrm{Al}_{2} \mathrm{O}_{3}{ }^{13}$ or $\mathrm{SnO}_{2}{ }^{14}$ overlayers to $\mathrm{Fe}_{2} \mathrm{O}_{3}$ photoanodes or the use of low-defect molybdenum chalcogenide layers in CIGS and CZTS solar cells. The buffer layers should be made of conductive, diamagnetic wide gap semiconductors that do not interfere with light absorption or introduce new recombination sites.

B. They must develop new ways to separate electrons and holes at interfaces. Because of the submicrometer size of many photocatalysts, the built-in electric fields in the light absorbers (space charge layers) are not effective for separating charge. Therefore, new ways are needed to increase charge separation. Recently, Krol's group reported tungsten gradient-doping as a way to generate a potential energy gradient inside of $\mathrm{BiVO}_{4}{ }^{15}$

Published: August 7, 2014 
This gradient moves photoelectrons toward the electrode and holes toward the solid-liquid interface. This is an elegant way to control charge separation, but it likely will be only usable in defect-tolerant semiconductors, such as $\mathrm{BiVO}_{4}$. For other semiconductors, the use of electron- or hole-selective coatings may be more suitable. Such coatings consist of transparent $n$ - or p-type metal oxides that selectively conduct electrons or holes and effect carrier separation at the interface. ${ }^{16}$ For example, n$\mathrm{SnO}_{2}$ underlayers on $\mathrm{BiVO}_{4}$ photoanodes have been found to boost the photocurrent by $300 \%{ }^{17}$ and p-type $\mathrm{NiO}$ layers on $\mathrm{Fe}_{2} \mathrm{O}_{3}$ improved the photovoltage by nearly $0.4 \mathrm{~V} .^{18}$ Many conducting $\mathrm{p}$ - and $\mathrm{n}$ - metal oxides can now be deposited from solution, and could potentially be applied to photocatalysts. ${ }^{19}$

C. They must develop proton-selective electrocatalysts to prevent the $\mathrm{H}_{2} / \mathrm{O}_{2}$ back-reaction (chemical shunting). For many proton reduction cocatalysts, electrochemical oxygen reduction proceeds rapidly, leading to the formation of superoxide and eventually water. This well-known back reaction limits the function of water splitting catalysts in the presence of $1 \mathrm{~atm}$ of oxygen/hydrogen. Possible solutions to this problem include the use of a $\mathrm{Rh} / \mathrm{Cr}_{2} \mathrm{O}_{3}$ cocatalyst on $\mathrm{GaN}: \mathrm{ZnO}$, which limits oxygen reduction at the $\mathrm{Rh}$ reduction site though a $\mathrm{Cr}_{2} \mathrm{O}_{3}$ barrier layer. ${ }^{20}$ Another one is the use of proton reduction cocatalysts with low activity for oxygen reduction, including $\mathrm{NiO}, \mathrm{Au}^{21}$ and $\mathrm{RuO}_{2}{ }^{8}$ and a third one is the use of dopants, like $\mathrm{Fe}^{3+}$, to suppress the oxygen reduction reaction on $\mathrm{Pt}^{22}$ The development of these and other proton selective electrocatalysts for uses in water splitting systems would be desirable.

In combination with new methods for the measurement of junction potentials, ${ }^{23,24}$ these guidelines should allow significant improvement of suspended water splitting photocatalyst technology and help define a path to inexpensive fuels from solar energy.

\section{Frank E. Osterloh*}

Department of Chemistry, University of

California-Davis, One Shields Avenue, Davis, California 95616, United States

\section{AUTHOR INFORMATION}

\section{Corresponding Author}

*E-mail: fosterloh@ucdavis.edu.

\section{Notes}

The authors declare no competing financial interest.

\section{ACKNOWLEDGMENTS}

F.E.O. is grateful for financial support from Research Corporation for Science Advancement (Scialog award) and from the National Science Foundation.

\section{REFERENCES}

(1) Walter, M. G.; Warren, E. L.; McKone, J. R.; Boettcher, S. W.; Mi, Q. X.; Santori, E. A.; Lewis, N. S. Solar Water Splitting Cells. Chem. Rev. 2010, 110, 6446-6473.

(2) Khaselev, O.; Turner, J. A. A Monolithic PhotovoltaicPhotoelectrochemical Device for Hydrogen Production via Water Splitting. Science 1998, 280, 425-427.

(3) Nozik, A. J. Photochemical Diodes. Appl. Phys. Lett. 1977, 30, 567-569.

(4) Pinaud, B. A.; Benck, J. D.; Seitz, L. C.; Forman, A. J.; Chen, Z. B.; Deutsch, T. G.; James, B. D.; Baum, K. N.; Baum, G. N.; Ardo, S.; et al. Technical and Economic Feasibility of Centralized Facilities for
Solar Hydrogen Production via Photocatalysis and Photoelectrochemistry. Energy Environ. Sci. 2013, 6, 1983-2002.

(5) James, B. D.; Baum, G. N.; Perez, J.; Baum, K. N. Technoeconomic Analysis of Photoelectrochemical (PEC) Hydrogen Production; http://www1.eere.energy.gov/hydrogenandfuelcells/ pdfs/pec_technoeconomic_analysis.pdf (accessed June 2014).

(6) Ohno, T.; Bai, L.; Hisatomi, T.; Maeda, K.; Domen, K. Photocatalytic Water Splitting Using Modified GaN:ZnO Solid Solution under Visible Light: Long-Time Operation and Regeneration of Activity. J. Am. Chem. Soc. 2012, 134, 8254-8259.

(7) Kato, H.; Asakura, K.; Kudo, A. Highly Efficient Water Splitting into $\mathrm{H}-2$ and O-2 over Lanthanum-Doped $\mathrm{NaTaO}_{3}$ Photocatalysts with High Crystallinity and Surface Nanostructure. J. Am. Chem. Soc. 2003, 125, 3082-3089.

(8) Kudo, A.; Miseki, Y. Heterogeneous Photocatalyst Materials for Water Splitting. Chem. Soc. Rev. 2009, 38, 253-278.

(9) Maeda, K. Photocatalytic Water Splitting Using Semiconductor Particles: History and Recent Developments. J. Photochem. Photobiol. C 2011, 12, 237-268.

(10) Osterloh, F. E. Inorganic Materials as Catalysts for Photochemical Splitting of Water. Chem. Mater. 2008, 20, 35-54.

(11) Osterloh, F. E. Inorganic Nanostructures for Photoelectrochemical and Photocatalytic Water Splitting. Chem. Soc. Rev. 2013, 42, 2294-2320.

(12) Yablonovitch, E.; Allara, D. L.; Chang, C. C.; Gmitter, T.; Bright, T. B. Unusually Low Surface Recombination Velocity on Silicon and Germanium Surfaces. Phys. Rev. Lett. 1986, 57, 249-252.

(13) Le Formal, F.; Tetreault, N.; Cornuz, M.; Moehl, T.; Gratzel, M.; Sivula, K. Passivating Surface States on Water Splitting Hematite Photoanodes with Alumina Overlayers. Chem. Sci. 2011, 2, 737-743.

(14) Spray, R. L.; McDonald, K. J.; Choi, K.-S. Enhancing Photoresponse of Nanoparticulate a- $\mathrm{Fe}_{2} \mathrm{O}_{3}$ Electrodes by Surface Composition Tuning. J. Phys. Chem. C 2011, 115, 3497-3506.

(15) Abdi, F. F.; Han, L. H.; Smets, A. H. M.; Zeman, M.; Dam, B.; van de Krol, R. Efficient Solar Water Splitting by Enhanced Charge Separation in a Bismuth Vanadate-Silicon Tandem Photoelectrode. Nat. Commun. 2013, 4, 2195.

(16) Dittrich, T.; Belaidi, A.; Ennaoui, A. Concepts of Inorganic Solid-State Nanostructured Solar Cells. Sol. Energy Mater. 2011, 95 (6), 1527-1536.

(17) Liang, Y. Q.; Tsubota, T.; Mooij, L. P. A.; van de Krol, R. Highly Improved Quantum Efficiencies for Thin Film $\mathrm{BiVO}_{4}$ Photoanodes. J. Phys. Chem. C 2011, 115, 17594-17598.

(18) Du, C.; Yang, X. G.; Mayer, M. T.; Hoyt, H.; Xie, J.; McMahon, G.; Bischoping, G.; Wang, D. W. Hematite-Based Water Splitting with Low Turn-On Voltages. Angew. Chem., Int. Ed. Engl. 2013, 52, 1269212695.

(19) Pasquarelli, R. M.; Ginley, D. S.; O’Hayre, R. Solution Processing of Transparent Conductors: From Flask to Film. Chem. Soc. Rev. 2011, 40, 5406-5441.

(20) Maeda, K.; Teramura, K.; Lu, D. L.; Saito, N.; Inoue, Y.; Domen, K. Roles of $\mathrm{Rh} / \mathrm{Cr}_{2} \mathrm{O}_{3}$ (core/shell) Nanoparticles Photodeposited on Visible-Light-Responsive $\left(\mathrm{Ga}_{1-x} \mathrm{Zn}_{x}\right)\left(\mathrm{N}_{1-x} \mathrm{O}_{x}\right)$ Solid Solutions in Photocatalytic Overall Water Splitting. J. Phys. Chem. C 2007, 111, 7554-7560.

(21) Iwase, A.; Kato, H.; Kudo, A. Nanosized Au Particles as an Efficient Cocatalyst for Photocatalytic Overall Water Splitting. Catal. Lett. 2006, 108, 6-9.

(22) Kato, H.; Sasaki, Y.; Wase, A.; Kudo, A. Role of Iron Ion Electron Mediator on Photocatalytic Overall Water Splitting Under Visible Light Irradiation Using Z-Scheme Systems. Bull. Chem. Soc. Jpn. 2007, 80, 2457-2464.

(23) Zhao, J.; Osterloh, F. E. Photochemical Charge Separation in Nanocrystal Photocatalyst Films - Insights from Surface Photovoltage Spectroscopy. J. Phys. Chem. Lett. 2014, 5, 782-786.

(24) Lin, F. D.; Boettcher, S. W. Adaptive Semiconductor/ Electrocatalyst Junctions in Water-Splitting Photoanodes. Nat. Mater. 2014, 13, 81-86. 